\title{
RESTRICTED DIOPHANTINE APPROXIMATION
}

\author{
R. T. WORLEY \\ (Received 15 July 1976, revised 25 January 1977) \\ Communicated by Jane Pitman
}

\begin{abstract}
The problem considered is that of approximating irrationals $\alpha$ by rationals $p / q$ where $p$ and $q$ avoid certain congruence classes mod $2^{k}$ for certain integers $k$. Results are obtained which give close bounds on a number $c$ such that $|\alpha-p / q|<c / q^{2}$ has infinitely many solutions where $p$ and $q$ can be expressed as the sum of three squares.
\end{abstract}

\section{Introduction}

Scott (1940) considered the problem of approximating real irrational numbers $\alpha$ by rationals $p / q$ where $p$ and $q$ are of given parity. Others (see section 47.6 of Lekkerkerker (1969)) have generalized this to the case $p \equiv r$ $(\bmod m), q \equiv s(\bmod m)$ where $(r, s, m)=1$. The problem to be discussed here is the complementary problem, namely the restriction $p \neq r(\bmod m)$, $q \not \equiv s(\bmod m)$, in the cases $m=4,8$ and 16 . We define $k(r, s, m)$ to be the infimum of the values of $k$ for which, for every real irrational $\alpha$, the inequality

$$
q|q \alpha-p|<k
$$

has infinitely many solutions in integers $p, q$ with $q>0$ satisfying

$$
p \not \equiv r(\bmod m), \quad q \not \equiv s(\bmod m) .
$$

In Section 2 we prove

THEOREM 1. For $m=4$ or 8 and $r$ and $s$ both odd

$$
k(r, s, m)=2 / \sqrt{ } 5
$$

and this is an attained minimum. 
THEOREM 2. $k(0,1,8)=7 \mathrm{~V} / 2 / 12$ is not attained.

It can be seen from the proofs that the constants $2 / \sqrt{ } 5$ and $7 \sqrt{ } 2 / 12$ are isolated, but in each case the irrationals $\alpha$ requiring the critical value of $k$ do not form a full equivalence class. The contrast between the two results is a little surprising. The method of proof used here enables $k(r, s, m)$ to be evaluated for most triples $(r, s, m)$, but it appears that a general proof giving $k(r, s, m)$ as a specific function of $r, s, m$ will not exist. Indeed the actual value of $k(r, s, m)$ seems almost to occur by accident. We shall also prove the following result.

THEOREM 3. For every irrational $\alpha>0$ there are infinitely many solutions of (1) with $k=27 / 2 \vee 145$ and $p$ and $q$ sums of three squares.

It should be noted that this value of $k$ is not shown to be best possible. However by combining this with the result of Theorem 1, remembering that numbers congruent to $7 \bmod 8$ cannot be written as the sum of three squares, we have the inequality:

COROLlaRY. The infimum $\kappa$ of the values of $k$ for which (1) has infinitely many solutions with $p$ and $q$ being sums of three squares satisfies

$$
2 / \vee 5 \leqq \kappa \leqq 27 / 2 \vee 145 \text {. }
$$

It will be noted that the proof of Theorem 1 also holds for the case $m=16, r s \equiv \pm 1 \bmod 16$ with little modification. The proof of Theorem 3 uses the well-known result that a positive integer can be written as the sum of three squares if and only if it is not of the form $4^{a}(8 b+7)$ for integers $a$ and $b$. At one stage much of the strength of this result is used, though for the rest of the proof the simple fact that numbers $4^{a}(8 b+7)$ are congruent to 0,4 or 7 $\bmod 8$ is sufficient.

The proofs of Theorems 1, 2 and 3 seek $\alpha$ for which the convergents (to the simple continued fraction of $\alpha$ ) violate the congruence condition. The convergents investigated include not only the primary convergents but also the secondary, and even "tertiary" convergents.

\section{2.}

In the proof of Theorems 1 and 2, we assume $\alpha>0$, for if $\alpha<0$ we just consider $-\alpha$ and replace $r$ by $-r \bmod m$. The proofs depend on the theory of simple continued fractions and we list the results needed from the standard theory in the following lemmas, proofs of which are essentially to be found in chapter 1 of Lang (1966). 
LeMma 1. Let $\alpha$ have continued fraction $\left[a_{0}, a_{1}, a_{2}, \cdots\right]$, and let $p_{n}$ and $q_{n}$ be defined inductively for $n \geqq 0$ by the equations

$$
\begin{array}{lll}
p_{0}=a_{0}, & p_{1}=a_{1} a_{0}+1, & p_{n}=a_{n} p_{n-1}+p_{n-2}, \\
q_{0}=1, & q_{1}=a_{1}, & q_{n}=a_{n} q_{n-1}+q_{n-2} .
\end{array}
$$

Furthermore, for $n \geqq 0$, define

$$
\begin{array}{ll}
P_{n}=p_{n+1}+p_{n}, & P_{n}^{\prime}=\left(a_{n+2}-1\right) p_{n+1}+p_{n}, \\
Q_{n}=q_{n+1}+q_{n}, & Q_{n}^{\prime}=\left(a_{n+2}-1\right) q_{n+1}+q_{n} .
\end{array}
$$

Then if $(p, q)=1$ and $q|q \alpha-p|<1$ either

(i) $(p, q)=\left(p_{n}, q_{n}\right)$ for some $n \geqq 0$, or

(ii) $(p, q)=\left(P_{n}, Q_{n}\right)$ or $\left(P_{n}^{\prime}, Q_{n}^{\prime}\right)$ for some $n \geqq 0$.

Furthermore (i) must hold if $q|q \alpha-p|<1 / 2$.

Lemma 2. Let $\alpha_{n}=\left[a_{n+1}, a_{n+2}, \cdots\right]$ and $\alpha_{n}^{\prime}=\left[0, a_{n}, a_{n-1}, \cdots, a_{1}\right]$. Then with the notation of Lemma 1

(i) $q_{n}\left|q_{n} \alpha-p_{n}\right|=1 /\left(\alpha_{n}+\alpha_{n}^{\prime}\right)=k_{n}$,

(ii) $Q_{n}\left|Q_{n} \alpha-P_{n}\right|=\left(\alpha_{n+1}-1\right)\left(\alpha_{n+1}^{\prime}+1\right) /\left(\alpha_{n+1}+\alpha_{n+1}^{\prime}\right)=k_{n}^{\prime}$, and

(iii) $Q_{n}^{\prime}\left|Q_{n}^{\prime} \alpha-P_{n}^{\prime}\right|=\left(\alpha_{n+1}+1-a_{n+2}\right)\left(\alpha_{n+1}^{\prime}+a_{n+2}-1\right) /\left(\alpha_{n+1}+\alpha_{n+1}^{\prime}\right)=k_{n}^{\prime \prime}$.

From the formula

$$
k_{n}^{\prime}=1-\left(2-\alpha_{n+1}+1 / \alpha_{n+1}^{\prime}\right) /\left(1+\alpha_{n+1} / \alpha_{n+1}^{\prime}\right)
$$

it is clear that $k_{n}^{\prime}>1$ unless

$$
\left[a_{n+2}, a_{n+3}, \cdots\right]<2+\left[a_{n+1}, a_{n}, \cdots, a_{1}\right] .
$$

Similarly from the formula

$$
k_{n}^{\prime \prime}=1-\left(2+\alpha_{n+2}-1 / \alpha_{n+2}^{\prime}\right) /\left(1+\alpha_{n+2} / \alpha_{n+2}^{\prime}\right)
$$

it is clear that $k_{n}^{\prime \prime}>1$ unless

$$
\left[a_{n+2}, a_{n+1}, \cdots, a_{1}\right]<2+\left[a_{n+3}, a_{n+4}, \cdots\right] .
$$

From these two formulae we also have $k_{n}^{\prime}+k_{n-1}^{\prime \prime}<2$.

Lemma 3. (i) Let $\alpha=\left[a_{0}, \cdots, a_{r}, a_{r+1}, \cdots\right]$, and $\beta=\left[a_{0}, \cdots, a_{r}, b_{r+1}, \cdots\right]$. Then $\alpha<\beta$ if $a_{r+1}<b_{r+1}$ and $r$ is odd or if $a_{r+1}>b_{r+1}$ and $r$ is even.

(ii) $\left[a_{11}, a_{1}, \cdots, a_{r}\right]<\left[a_{0}, a_{1}, \cdots, a_{r}, \cdots\right]<\left[a_{0}, a_{1}, \cdots, a_{r}+1\right]$ if $r$ is even, otherwise the reverse inequalities hold.

We can use these results to show that, for $r s$ odd, $k(r, s, 8) \geqq 2 / \sqrt{ } 5$ as follows. 
LEMMA 4. Let $r s$ be odd, and let $\alpha=\left[a_{0}, a_{1}, \cdots\right]$ be such that for some integer $l$ the following conditions hold.

(i) $a_{n}=4$ for $n \geqq l+2$,

(ii) $p_{t} \equiv r(\bmod 8)$ and $q_{1+1} \equiv s(\bmod 8)$, and

(iii) both $q_{1}$ and $p_{1+1}$ are even.

Then there are infinitely many pairs $(p, q)$ of integers with $q>0$ satisfying (1) and (2) with $m=8$ and $k=2 / \sqrt{ } 5$, but there are only finitely many pairs when $k<2 / \vee 5$.

Proof. Let $p$ and $q$ satisfy (1) with $k \leqq 2 / \sqrt{ } 5$. Then by Lemma 1 one of the following must be true.

(i) $(p, q)=\left(p_{n}, q_{n}\right)$ and $q|q \alpha-p|=k_{n}$,

(ii) $(p, q)=\left(P_{n}, Q_{n}\right)$ and $q|q \alpha-p|=k_{n}^{\prime}$,

(iii) $(p, q)=\left(P_{n}^{\prime}, Q_{n}^{\prime}\right)$ and $q|q \alpha-p|=k_{n}^{\prime \prime}$,

(iv) $(p, q)=\left(t p_{n}, t q_{n}\right)$ and $q|q \alpha-p|=t^{2} k_{n} \geqq 4 k_{n}$.

Now $[4, \dot{4}]=2+\sqrt{ } 5$, so since

$$
\lim _{n \rightarrow \infty} k_{n}^{\prime}=\lim _{n \rightarrow \infty} k_{n}^{\prime \prime}=4 \lim _{n \rightarrow \infty} k_{n}=2 / \sqrt{ } 5
$$

it is clear that with $k<2 / \sqrt{ } 5$ equations (1) and (2) can have at most finitely many solutions arising from cases (ii), (iii) and (iv). In addition since for $\boldsymbol{n} \geqq l$ we have either $p_{n} \equiv r(\bmod 8)$ or $q_{n} \equiv s(\bmod 8)$ only finitely many solutions of (1) and (2) can arise from case (i) when $k<2 / \sqrt{ } 5$. To complete the proof of the lemma it remains to show that $(p, q)=\left(2 p_{n}, 2 q_{n}\right)$ satisfies (1) and (2) with $k=2 / \sqrt{ } 5$ infinitely often. This is a trivial consequence of the fact that for $n \geqq l, 4 k_{n}<2 / \sqrt{ } 5$ if $\alpha_{n}^{\prime}>\sqrt{ } 5-2$ and $4 k_{n+1}<2 / \sqrt{ } 5$ if $\alpha_{n}^{\prime}<\sqrt{ } 5-2$.

It must of course be noted that it is possible to construct, for each choice of odd $r$ and $s$, an irrational satisfying these conditions: for example for $(r, s)=(1,1)$ we can take $\alpha=[8, \dot{4}]$, for $(r, s)=(1,3)$ we can take $\alpha=$ $[0,1,1,1, \dot{4}]$. Essentially all that is required is to choose that one of $r s+1$, $r s-1$ that is divisible by 4 and let $a b$ be a factorization of that number into even factors. Then $r / a, b / s$ are consecutive convergents $p_{l} / q_{1}, p_{1+1} / q_{1+1}$ of some finite continued fraction $\left[a_{0}, a_{1}, \cdots, a_{t+1}\right]$ which can be found by reversing the continued fraction algorithm. Setting $\alpha=\left[a_{0}, a_{1}, \cdots, a_{l+1}, \dot{4}\right]$ satisfies the requirements.

It can also be verified that if $r s \equiv \pm 1 \bmod 16$ and we choose $a$ and $b$ both divisible by 4 such that $a b=r s \pm 1$ then the above procedure yields an $\alpha$ that satisfies conditions (i), (ii) and (iii) of the lemma where the congruences in (ii) are now modulo 16, and in (iii) both $q_{1}$ and $p_{i+1}$ are divisible by 4 . Following the proof of the lemma through yields $k(r, s, 16) \geqq 2 / \vee 5$ for $r s \equiv \pm 1 \bmod 16$. 
To show $k(r, s, 8)=2 / \sqrt{ } 5$ for $r s$ odd it remains to show the following result.

Lemma 5. Let $r$ be odd, $m=4$ and $k=2 / \sqrt{ } 5$. Then equations (1) and (2) have infinitely many solutions.

Proof. If $\alpha=\left[a_{0}, a_{1}, a_{2}, \cdots\right]$ then one of the following possibilities must occur.

(i) $a_{n} \geqq 5$ for infinitely many $n$,

(ii) $a_{n} \leqq 3$ for all sufficiently large $n$,

(iii) $a_{n}=4$ for all sufficiently large $n$,

(iv) $a_{n} \leqq 4$ for all sufficiently large $n$ and either

(a) $a_{n-1}=1$ or $a_{n+1}=1$ for infinitely many $n$ for which $a_{n}=4$, or

(b) $a_{n-1} \geqq 2$ and $a_{n+1} \geqq 2$ for all sufficiently large $n$ for which $a_{n}=4$ and $a_{n+1} \leqq 3$ for infinitely many of these $n$.

The result for case (iii) follows by taking $(p, q)=\left(2 p_{n}, 2 q_{n}\right)$ as in the proof of Lemma 4, while in cases (i), (iv)(a) and (iv)(b) we only need to take $(p, q)=\left(2 p_{n}, 2 q_{n}\right)$ for infinitely many $n$ for which $k_{n}<1 / 2 \sqrt{ } 5$. In case (i) we have $k_{n}<1 / 5$ for the infinitely many $n$ for which $a_{n+1} \geqq 5$, in case (iv)(a) we have

$$
k_{n}^{-1}>4+[0,1,1]+[0,4,1]=4.7
$$

infinitely often, and in case (iv)(b) we have

$$
k_{n}^{-1}>[4,3,1,5]+[0,4,2,5]>4.48
$$

infinitely often. We are therefore left with case (ii).

Let $l$ be chosen so that $a_{n} \leqq 3$ for $n \geqq l$. For $n>l$

$$
\alpha_{n}+\alpha_{n}^{\prime}>[1,3,1]+[0,3,1]=1.5
$$

and setting $(p, q)=\left(p_{n}, q_{n}\right)$ gives infinitely many solutions to (1) and (2) with $k=2 / \sqrt{ } 5$ unless $\left(p_{n}, q_{n}\right)$ does not satisfy (2) for all $n$ greater than some $l_{0}$. We therefore assume from now on that $l^{\prime}(\geqq l)$ is such that for all $n>l^{\prime}$ either $p_{n} \equiv r(\bmod 4)$ or $q_{n} \equiv s(\bmod 4)$.

For the present we shall also assume that $r=s=1$ and denote by $h$ an integer greater than $l^{\prime}+6$. We let $T_{h}$ denote the matrix

$$
\left(\begin{array}{cc}
p_{h} & q_{h} \\
p_{h+1} & q_{h+1}
\end{array}\right)
$$

where each entry has been reduced mod 4 to lie in $\{0,1,2,3\}$. Using the conditions (a) $p_{h}=1(\bmod 4)$ or $q_{h} \equiv 1(\bmod 4)$ and $(b)\left|p_{h} q_{h+1}-p_{h+1} q_{h}\right|=1$, this latter being a standard property of continued fractions, either $S_{i}=T_{h}$ or 
$S_{i}^{*}=T_{h}$ where $S_{i}^{*}$ denotes $S_{i}$ with transposed columns and $S_{i}$ denotes one of the following matrices:

$$
\begin{aligned}
& S_{1}=\left(\begin{array}{ll}
1 & 0 \\
1 & 1
\end{array}\right) \quad S_{2}=\left(\begin{array}{ll}
1 & 1 \\
1 & 0
\end{array}\right) \quad S_{3}=\left(\begin{array}{ll}
1 & 1 \\
1 & 2
\end{array}\right) \quad S_{4}=\left(\begin{array}{ll}
1 & 2 \\
1 & 1
\end{array}\right) \\
& S_{5}=\left(\begin{array}{ll}
1 & 2 \\
1 & 3
\end{array}\right) \quad S_{6}=\left(\begin{array}{ll}
1 & 3 \\
1 & 2
\end{array}\right) \quad S_{7}=\left(\begin{array}{ll}
1 & 3 \\
1 & 0
\end{array}\right) \quad S_{8}=\left(\begin{array}{ll}
1 & 0 \\
1 & 3
\end{array}\right) \\
& S_{9}=\left(\begin{array}{ll}
1 & 0 \\
2 & 1
\end{array}\right) \quad S_{10}=\left(\begin{array}{ll}
1 & 0 \\
3 & 1
\end{array}\right) \quad S_{11}=\left(\begin{array}{ll}
1 & 3 \\
2 & 1
\end{array}\right) \quad S_{12}=\left(\begin{array}{ll}
1 & 2 \\
0 & 1
\end{array}\right) \\
& S_{13}=\left(\begin{array}{ll}
1 & 3 \\
0 & 1
\end{array}\right) \quad S_{14}=\left(\begin{array}{ll}
1 & 2 \\
3 & 1
\end{array}\right) \quad S_{15}=\left(\begin{array}{ll}
1 & 0 \\
0 & 1
\end{array}\right) \quad S_{16}=\left(\begin{array}{ll}
1 & 2 \\
2 & 1
\end{array}\right) \text {. }
\end{aligned}
$$

Since $T_{h+1}=\left(\begin{array}{cc}0 & 1 \\ 1 & a_{h+2}\end{array}\right) T_{h}(\bmod 4)$ and $a_{h+2} \leqq 3$ we can form the following graph, where $S_{i} \stackrel{j}{\rightarrow} S_{i}$, denotes that if $T_{h}$ is $S_{i}$ or $S_{i}^{*}$ then $T_{h+1}=S_{i}$. or $S_{i}^{*}$. if $a_{h+2}=j$.

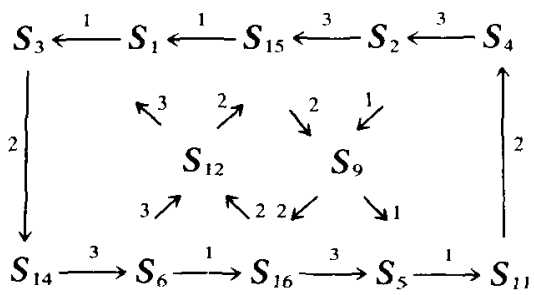

It will be noted that $S_{7}, S_{8}, S_{10}$ and $S_{13}$ disappear from consideration. Using Lemma 3 we find that $\alpha_{h+1} \leqq[2,3,3,2,2, \cdots]$ and $\alpha_{h+1}^{\prime} \leqq[0,1,3,2,2,1, \cdots]$ when $T_{h}=S_{3}$ or $S_{3}^{*}$, and $\alpha_{h+1} \leqq[2,3,3,1,1, \cdots]$ and $\alpha_{h+1}^{\prime} \leqq[0,1,3,1,3,2, \cdots]$ when $T_{h}=S_{11}$ or $S_{11}^{*}$. Thus since $k_{h}^{\prime}$ is a maximum when both $\alpha_{h+1}$ and $\alpha_{h+1}^{\prime}$ are a maximum we have that $k_{h}^{\prime}<.755$ whenever $T_{h} \in \mathscr{S}=\left\{S_{3}, S_{3}^{*}, S_{11}, S_{11}^{*}\right\}$. Now $(p, q)=\left(P_{h}, Q_{h}\right)$ satisfies (2) whenever $T_{h} \in \mathscr{P}$, and so if $T_{h} \in \mathscr{P}$ for infinitely many $h$ we get infinitely many solutions of (1) and (2) with $k=2 / \vee 5$. We therefore assume that $T_{h} \in \mathscr{S}$ for only finitely many $h$, that is for $h \geqq h_{0}, T_{h}$ or $T_{h}^{*}$ cycles around the subgraph

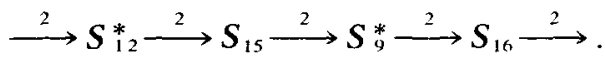

Since $[\dot{2}]=1+\sqrt{ } 2$ we have $\lim _{h \rightarrow x} k_{h}^{\prime}=2^{-\frac{1}{2}}<0.71$. This means that, since $T_{h}=S_{16}$ or $S_{16}^{*}$ implies that $(p, q)=\left(P_{h}, Q_{h}\right)$ satisfies (2), we again have infinitely many solutions of (1) and (2) with $k=2 / \mathrm{V} 5$. 
This completes the discussion of case (ii) for $r=s=1$. The same proof applies for the other three cases on letting $T_{h}$ denote, respectively, the matrix

$$
\left(\begin{array}{cc}
p_{h} & -q_{h} \\
p_{h+1} & -q_{h+1}
\end{array}\right), \quad\left(\begin{array}{cc}
-p_{h} & -q_{h} \\
-p_{h+1} & -q_{h+1}
\end{array}\right),\left(\begin{array}{cc}
-p_{h} & q_{h} \\
-p_{h+1} & q_{h+1}
\end{array}\right)
$$

reduced $\bmod 4$ in the cases $(r, s)=(1,3),(3,3),(3,1)$.

Theorem 1 now follows as a consequence of Lemma 4 and Lemma 5. The proof of Theorem 2 is basically similar, though it is more complicated since $(2 p, 2 q)$ will not always satisfy (2). To save repetition we shall from now on take $r=0, s=1, m=8$ in (2).

LEMMA 6. If, for some $l>0, a_{n}=1$ for $n \geqq l$ then $q|q \alpha-p|<0.82$ has infinitely many solutions satisfying (2).

Proof. If $n \geqq l$ then one of the pairs $\left(p_{h}, q_{n}\right), h=n, n+1, n+2$ satisfies (2), for it is easily verified that the equations

(a) $p_{n+1}+p_{n} \equiv 0(\bmod 8)$ or $q_{n+1}+q_{n} \equiv 1(\bmod 8)$,

(b) $p_{n+1} \equiv 0(\bmod 8)$ or $q_{n+1} \equiv 1(\bmod 8)$,

(c) $p_{n} \equiv 0(\bmod 8)$ or $q_{n} \equiv 1(\bmod 8)$,

(d) $\left|p_{n} q_{n+1}-p_{n+1} q_{n}\right|=1$

have no solution. Now $[i]=\frac{1}{2}(1+\sqrt{ } 5)$ and $k_{n} \rightarrow 1 / \sqrt{ } 5$, so clearly $q|q \alpha-p|<$ 0.82 must have infinitely many solutions $(p, q)=\left(p_{n}, q_{n}\right)$ satisfying (2).

In the following Lemmas $7-10$ we mean by assumptions (A) the following: $q|q \alpha-p|<.82$ has finitely many solutions satisfying (2), and $l$ is chosen such that none of the solutions is of the form $\left(d p_{n}, d q_{n}\right)$ with $n \geqq l$. In the proof of the lemmas we make frequent use of the standard result $\left|p_{n+1} q_{n}-p_{n} q_{n+1}\right|=1$.

Lemma 7. Under assumptions (A) let $h>l$ be such that $a_{h+1} \geqq 2$, $q_{h} \equiv q_{h+1} \equiv 1 \bmod 8$, and $p_{h+1} \neq 0 \bmod 8$. Then

(i) $a_{h+2} \leqq 4$ and $p_{h+2} \equiv 0 \bmod 8$ if $p_{h+1} \equiv 0 \bmod 4$, or

(ii) $a_{h+2}=8$ if $p_{h+1} \equiv 4 \bmod 8$.

Proof. (i) Suppose $p_{h+1} \not \equiv 0 \bmod 4$. We have $a_{h+2} \leqq 4$ immediately, for $\left(2 p_{h+1}, 2 q_{h+1}\right)$ satisfies (2), implying $4 k_{h+1} \geqq .82$. Now suppose that $p_{h+2} \neq 0$ mod 8. Since $q_{h+2} \equiv a_{h+2}+1$ it is clear that $\left(p_{h+2}, q_{h+2}\right)$ satisfies (2), so $k_{h+2} \geqq .82$. This is impossible unless $a_{h+2}=4, a_{h+3}=1$ and $a_{h+4} \geqq 50$. But now $\left(p_{h+3}, q_{h+3}\right)$ satisfies (2) and $k_{h+3}<1 / 50$, contrary to assumptions (A). This proves that $p_{\mathrm{h}+2} \equiv 0 \bmod 8$.

(ii) Suppose $p_{h+1} \equiv 4 \bmod 8$. Then $a_{h+2} \leqq 10$, for $9 k_{h+1} \geqq .82$ since $\left(3 p_{h+1}, 3 q_{h+1}\right)$ satisfies (2). Now suppose $a_{h+2} \neq 8$. Then $\left(p_{h+2}, q_{h+2}\right)$ satisfies (2), so $k_{h+2} \geqq .82$, from which it follows, since $\alpha_{h+2}^{\prime}>1 / 11$, that $a_{h+3}=1$ and 
$a_{h+4} \geqq 7$. But now $\left(2 p_{h+3}, 2 q_{h+3}\right)$ satisfies (2) and $4 k_{h+3}<4 / 7$ contradicting (A). This proves that $a_{h+2}=8$, as desired.

COROLlary. Under the assumptions of Lemma 7 , if $p_{h+1} \equiv 4 \bmod 8$, then $a_{h+3} \leqq 4$ and $p_{h+3} \equiv 0 \bmod 8$.

Proof. Apply the lemma with $h$ replaced by $h+1$, since not both $p_{h+1}$ and $p_{h+2}$ can be even.

LemmA 8. Under assumptions (A) let $h \geqq l$ be such that $a_{h+2} \geqq 2$. Then there exists $j \geqq h$ such that either $p_{i} \equiv 0 \bmod 8$ and $q_{j+1} \equiv 1 \bmod 8$ or $q_{j} \equiv 1 \bmod$ 8 and $p_{i+1} \equiv 0 \bmod 8$.

Proof. Since $k_{h}<1 / 2$ we either have $p_{h}^{\prime} \equiv 0 \bmod 8$ or $q_{h} \equiv 1 \bmod 8$. Suppose firstly that $p_{h} \equiv 0 \bmod 8$. We show that we may take $j=h$, for suppose to the contrary that $q_{h+1} \neq 1 \bmod 8$. Then $\left(p_{h+1}, q_{h+1}\right)$ satisfies (2), so $k_{h+1} \geqq .82, a_{h+2}=1$ and $a_{h+2} \geqq 4$. Now $\left(2 p_{h+2}, 2 q_{h+2}\right)$ satisfies (2), so $4 k_{h+2} \geqq .82$ and $a_{h+3} \leqq 4$, i.e. $a_{h+3}=4$. Furthermore as $k_{h+2}<.82$ we have $q_{h+2} \equiv 1 \bmod 8$ since $p_{h+2}$ is odd. This implies $q_{h+1}$ is even, so $\left(p_{h+3}, q_{h+3}\right)$ satisfies (2). For $k_{h+3} \geqq .82$ we require $a_{h+4}=1$ and $a_{h+5} \geqq 50$. However $2 p_{h+4} \equiv 4 \bmod 8$ since $p_{h+4} \equiv 6 p_{h+1} \bmod 8$, so $\left(2 p_{h+4}, 2 q_{h+4}\right)$ satisfies (2) and $4 k_{h+4}<.82$. This contradiction shows that we may take $j=h$, as claimed.

We now suppose that $q_{h} \equiv 1 \bmod 8$. Since we could set $j=h$ if $p_{h+1} \equiv 0$ $\bmod 8$, and $j=h+1$ or $j=h+2$ if $q_{h+1} \equiv 1 \bmod 8$ (by Lemma 7 and its corollary) we assume $p_{h+1} \not \equiv 0 \bmod 8$ and $q_{h+1} \neq 1 \bmod 8$. Now $\left(p_{h+1}, q_{h+1}\right)$ satisfy (2), so $k_{h+1} \geqq .82, a_{h+2}=1, a_{h+1} \geqq 4$ and $a_{h+3} \geqq 4$. Since $k_{h+2}<.82$ it is clear that $\left(p_{h+2}, q_{h+2}\right)$ cannot satisfy $(2)$. We assume $q_{h+2} \equiv 1 \bmod 8$, for $p_{h+2} \equiv 0$ mod 8 would imply we could take $j=h+2$ by the first part of this proof. Repeating the above argument with $h$ replaced by $h+2$ we can take $a_{h+4}=1$, $a_{h+5} \geqq 4$ and $q_{h+4} \equiv 1 \bmod 8$. Now $q_{h+1} \equiv q_{h+2}-q_{h} \equiv 0 \bmod 8$ and $q_{h+3} \equiv 0 \bmod$ 8 similarly, so $a_{h+3} \equiv 0 \bmod 8$. In particular $a_{h+3} \geqq 8$ and $4 k_{h+2}<.82$. Thus $\left(2 p_{h+2}, 2 q_{h+2}\right)$ cannot satisfy (2), so $p_{h+2} \equiv 0 \bmod 4$. Similarly we can show $p_{h+4} \equiv 0 \bmod 4$, so $p_{h+3} \equiv p_{h+4}-p_{h+2}$ is also even. However this contradicts $\left|p_{h+2} q_{h+3}-p_{h+3} q_{h+2}\right|=1$, and this is sufficient to prove the lemma.

Lemma 9. Under assumptions (A) let $h>l$ be such that $q_{h} \equiv 1 \bmod 8$ and $p_{h+1} \equiv 0 \bmod 8$. Then $a_{h+2} \equiv 0 \bmod 8$ and $q_{h+2} \equiv 1 \bmod 8$.

Proof. Suppose $a_{h+2} \not \equiv 0 \bmod 8$. Then $\left(p_{h+2}, q_{h+2}\right)$ satisfy (2) since $p_{h} q_{h+1}$ is odd, so $k_{h+2} \geqq .82, a_{h+3}=1, a_{h+4} \geqq 4$ and $a_{h+2} \geqq 4$. Now $p_{h+3}$ is odd, but $\left(p_{h+3}, q_{h+3}\right)$ doesn't satisfy (2) since $k_{h+3}<.82$, so $q_{h+3} \equiv 1 \bmod 8$. This implies that $\left(2 p_{h+3}, 2 q_{h+3}\right)$ satisfies (2). We therefore have $a_{h+4}=4$ and $a_{h+5} \geqq 2$ in order to have $4 k_{h+4} \geqq .82$. Since $q_{h+4}$ is even we have $\left(p_{h+4}, q_{h+4}\right)$ satisfying (2) 
and $k_{h+4}<1 / 2$. This contradiction implies that $a_{h+2} \equiv 0 \bmod 8$ as desired.

LeMMA 10. Under assumptions (A) let $h>l$ be such that $p_{h} \equiv 0 \bmod 8$ and $q_{h+1} \equiv 1 \bmod 8$. Then $a_{h+3} \geqq 2$ and $a_{h+2}=4$.

PROOF. Since both $p_{h+1}$ and $q_{h}$ are odd, $\left(2 p_{h+1}, 2 q_{h+1}\right)$ satisfies (2), so $a_{h+2} \leqq 4$ to ensure $4 k_{h+1} \geqq .82$. Now if $a_{h+2} \neq 4$ we have $k_{h+3}<.82$, so $q_{h+2} \equiv 1$ since $p_{h+2} \not \equiv 0 \bmod 8$. By Lemma 7 we therefore have, since $a_{h+2}$ must be even, that $p_{h+3} \equiv 0 \bmod 8$. However since $a_{h+2}=2$ is even $p_{h+3}$ is odd, so $p_{h+3} \equiv 0$ $\bmod 8$ is impossible. This leaves only the possibility that $a_{h+2}=4$. Now if $a_{h+3}=1$ we would have $p_{h+3}$ odd, $q_{h+3}$ even, so $\left(p_{h+3}, q_{h+3}\right)$ satisfies (2) yet $k_{h+3}<.82$. This contradiction shows $a_{h+3} \geqq 2$ as desired.

LEMMA 11. Under the same assumptions as Lemma $10 a_{h+3}=8, q_{h} \equiv 5$ $\bmod 8, q_{h+2} \equiv q_{h+3} \equiv 1 \bmod 8, a_{h+4}=4$ and $p_{h+4} \equiv 0 \bmod 8$.

Proof. From Lemma 10 we have $k_{h+2}<1 / 2$, so either $p_{h+2} \equiv 0 \bmod 8$ or $q_{h+2} \equiv 1 \bmod 8$. The first alternative cannot hold, since $p_{h+2}$ is four times an odd number, so the second alternative holds. Hence $q_{h} \equiv 1-4 \equiv 5 \bmod 8$, as desired. Furthermore, by Lemma 7, $a_{h+3}=8$ and again by Lemma 7 , since $p_{h+3}$ is odd, $p_{h+4} \equiv 0 \bmod 8$ and $a_{h+4} \leqq 4$. However $p_{h+4} \equiv 4+a_{h+4} p_{h+3}$ where $p_{h+3}$ is odd certainly cannot hold for $a_{h+4}<4$ and $p_{h+4} \equiv 0 \bmod 8$, so we have $a_{h+4}=4$.

Corollary. Assumptions (A) imply that for some $j>l, p_{i+4 n} \equiv 0 \bmod 8$, $q_{i+4 n} \equiv 5 \bmod 8, q_{j+1+4 n} \equiv q_{i+2+4 n} \equiv q_{j+3+4 n} \equiv 1 \bmod 8, p_{i+4 n+2} \equiv 4 \bmod 8$, $p_{i+1+2 n} \equiv p_{i+1}, a_{j+2 n}=4$ and $a_{j+2 n+1}=8$ for all $n \geqq 0$.

Proof. This is just a straightforward application of Lemmas 6,8 and 9 followed by repeated application of Lemmas 11 and 9 .

Now that we have isolated the critical case all that remains is to prove that this case is critical.

Lemma 12. Let $\alpha=\left[a_{0}, a_{1}, \cdots\right]$ be such that for some integer $j, q_{j} \equiv 5$ $\bmod 8, p_{i} \equiv 0 \bmod 8, q_{i+1} \equiv 1 \bmod 8, a_{j+2 n}=4$ for $n \geqq 0$ and $a_{j+2 n+1}=8$ for $n \geqq 0$. Then (1) and (2) have infinitely many solutions for $k>7 \sqrt{ } 2 / 12$. However if $\left[8, a_{j}, \cdots, a_{1}\right]<[\overline{8,4}]$ and $k=7 \sqrt{ } 2 / 12$ there are only finitely many solutions.

Proof. By Lemmas 1 and 2 we only need to investigate as possibilities for $(p, q)$ the pairs $\left(p_{h}, q_{h}\right),\left(P_{h}, Q_{h}\right),\left(P_{h}^{\prime}, Q_{h}^{\prime}\right)$ and $\left(d p_{h}, d q_{h}\right)$. We only consider $h \geqq j$ since we are looking for infinitely many solutions. There are a number of cases to be considered. 
(i) The pairs $\left(p_{h}, q_{h}\right)$. These never satisfy (2).

(ii) The pairs $\left(2 p_{h}, 2 q_{h}\right)$. These satisfy (2) only for $h=j+n$ with $n$ odd. In this case

$$
4 k_{h}=4 /\left([\overline{4,8}]+\left[0,8, \cdots, a_{1}\right]\right)>16 / 17 .
$$

(iii) The pairs $\left(d p_{h}, d q_{h}\right)$ for $d \geqq 3$. In this case $9 k_{h}>1$.

(iv) The pairs $\left(P_{h}, Q_{h}\right)$ for $h=j+n$ with $n$ odd. In this case $k_{h}^{\prime}>1$.

(v) The pairs $\left(P_{h}^{\prime}, Q_{h}^{\prime}\right)$ for $h=j+n$ with $n$ even. In this case $k_{h}^{\prime \prime}>1$.

(vi) The pairs $\left(P_{h}, Q_{h}\right)$ for $h=j+n$ with $n$ even. In this case

$$
k_{h}^{\prime}=1-\left(2-(\overline{4,8})+\left[8, \cdots, a_{1}\right]\right) /\left(1+[\overline{4,8}]\left[8, \cdots, a_{1}\right]\right)
$$

tends to $7 \sqrt{ } 2 / 12$ as $h \rightarrow \infty$. Furthermore $k_{h}^{\prime}<7 \sqrt{ } 2 / 12$ if and only if $\left[8, \cdots, a_{1}\right]>[\overline{8,4}]$, that is $\left[8, a_{i}, \cdots, a_{1}\right]>[\overline{8,4}]$.

(vii) The pairs $\left(P_{h}^{\prime}, Q_{h}^{\prime}\right)$ for $h=j+n$ with $n$ odd. In this case $k_{h}^{\prime \prime} \rightarrow 7 \vee 2 / 12$ as $h \rightarrow \infty$ and $k_{h}^{\prime \prime}<7 \vee 2 / 12$ if and only if $\left[4,8, \cdots, a_{1}\right]<[\overline{4,8}]$, that is $\left[8, a_{i}, \cdots, a_{1}\right]>[\overline{8,4}]$. The lemma follows immediately from the results of these cases.

Theorem 2 now follows immediately from the above lemma and corollary on observing that the hypotheses of Lemma 12 are satisfied by $\alpha=[4,1, \overline{4,8}]$.

\section{3.}

The proof of Theorem 3 depends on the result that numbers not of the form $4^{a}(8 k+7)$ can be written as the sum of three squares. The first point to note is that $27 / 2 \vee 145=1.121 \ldots>1$ and we therefore need a modified form of Lemma 1.

Lemma 13. Let $\alpha$ be as in Lemma 1 and let $p$ and $q$ be integers such that $(p, q)=1$ and $1<q|q \alpha-p|<2$. Then either (ii) of Lemma 1 holds or for some integer $n$

(iv) $(p, q)=\left(i p_{n}+p_{n-1}, i q_{n}+q_{n-1}\right)$ with $0<i<a_{n+1}$, or

(v) $(p, q)=\left((2 i+1) p_{n}+2 p_{n-1},(2 i+1) q_{n}+2 q_{n-1}\right)$ with $0 \leqq i<a_{n+1}$.

Proof. Since $(p, q) \neq\left(p_{n}, q_{n}\right)$ for any $n$ (Lemma 2$)$ there either exists $n$ such that

$$
p_{n-1} / q_{n-1}<p / q<p_{n+1} / q_{n+1}<\alpha<p_{n} / q_{n}
$$


or there exists $n$ such that the above holds with all inequalities reversed. We consider the first possibility only, as the second requires a similar argument. From the definition of $p_{n+1}, q_{n+1}$ there exists $i, 0 \leqq i<a_{n+1}$, such that

$$
\frac{{ }^{(i)} P_{n}}{{ }^{(i)} Q_{n}}<\frac{p}{q} \leqq \frac{{ }^{(i+1)} P_{n}}{{ }^{(i+1)} Q_{n}}<\alpha
$$

where ${ }^{(i)} P_{n}, \quad{ }^{(i)} Q_{n} \quad$ denote $i p_{n}+p_{n-1}, \quad i q_{n}+q_{n-1}$ respectively. Since ${ }^{(i+1)} P_{n}^{(i)} Q_{n}-{ }^{(i)} P_{n}^{(i+1)} Q_{n}=1$ we have

$$
\frac{1}{{ }^{(i)} Q_{n}{ }^{(i+1)} Q_{n}}=\frac{(i+1)}{{ }^{(i+1)} Q_{n}}-\frac{{ }^{(i)} P_{n}}{{ }^{(i)} Q_{n}} \geqq \frac{s}{q^{(i)} Q_{n}}
$$

where $s={ }^{(i)} Q_{n} p-{ }^{(i)} P_{n} q>0$. In addition

$$
2 q^{-2}>|\alpha-p / q|>r / q^{(i+1)} Q_{n}
$$

where $r={ }^{(i+1)} P_{n} q-{ }^{(i+1)} Q_{n} p \geqq 0$. Combining (3) and (4) gives

$$
r s^{(i+1)} Q_{n}<r q<2^{(i+1)} Q_{n}
$$

and so $r s<2$. The proof of the lemma is now complete on observing that (iv) holds if $r=0$ and ( $v$ ) holds if $r=s=1$.

We note in passing that if (iv) holds then

$$
q|q \alpha-p|=\left(\alpha_{n}-i\right)\left(\alpha_{n}^{\prime}+i\right) /\left(\alpha_{n}+\alpha_{n}^{\prime}\right), \quad={ }^{(i)} k_{n} \text { say, }
$$

and if $(v)$ holds then

$$
q|q \alpha-p|=\left(2 \alpha_{n}-(2 i+1)\right)\left(2 \alpha_{n}^{\prime}+(2 i+1)\right) /\left(\alpha_{n}+\alpha_{n}^{\prime}\right), \quad={ }^{(i)} k_{n}^{\prime} \quad \text { say. }
$$

The following lemma is required to show that certain irrational $\alpha$ can be approximated by a quotient of sums of three squares sufficiently well.

LemMA 14. Let $\alpha=\left[a_{0}, a_{1}, \cdots\right]$ be such that for some integer $l$ the following conditions hold.

(i) $p_{t-1} \equiv 0 \bmod 8, q_{t-1} \equiv 1 \bmod 8, p_{i} \equiv q_{i} \equiv 7 \bmod 8$,

(ii) $a_{1+3 m+1}=2, a_{1+3 m+2}=7$ and $a_{1+3 m}=1$ for all integers $m \geqq 0$.

Then there exist infinitely many pairs $(p, q)$ of integers with $q>0$ satisfying (1) with $k=27 / 2 \vee 145\left(=k^{*}\right.$, say) and neither $p$ nor $q$ congruent to 0,4 or 7 mod 8 , but there are finitely many such pairs if $k<k^{*}$.

Proof. For convenience we shall let (c) denote the condition: neither $p$ nor $q$ is congruent to 0,4 or 7 . Let $(p, q)$ satisfy (1) with $k \leqq k^{*}$ and condition (c). Then by Lemmas 1 and 14 either

(i) $(p, q)=\left(t p_{n}, t q_{n}\right)$ for some integer $t$,

(ii) $(p, q)=\left({ }^{(i)} P_{n},{ }^{(i)} Q_{n}\right)$ with $0<i<a_{n+1}$, or 
(iii) $(p, q)=\left({ }^{(i)} P_{n}+{ }^{(i+1)} P_{n},{ }^{(i)} Q_{n}+{ }^{(i+1)} Q_{n}\right)$ with $0 \leqq i<a_{n+1}$.

We therefore need to consider $t^{2} k_{n},{ }^{(i)} k_{n}$ and ${ }^{(i)} k_{n}^{\prime}$. For convenience we denote by $\lim _{3} k_{1}$ the limit

$$
\lim _{m \rightarrow \infty} k_{l+3 m}
$$

with similar notation for ${ }^{(i)} k_{l},{ }^{(i)} k_{l}^{\prime}$. We look at three cases.

(a) Consider $n \equiv l+2 \bmod 3$. Then possibility (ii) is vacuous and $t$ must be at least 2 in case (i).

Since $\lim _{3} k_{l+2}=15 / 2 \mathrm{~V} 145$ and $\lim _{3}{ }^{(0)} k_{l+2}^{\prime}=37 / 2 \vee 145$ there are clearly only finitely many possibilities for $(p, q)$ in this case.

(b) Consider $n \equiv l \bmod 3$. Then $t$ must be at least 2 in case (i) and case (ii) is excluded since $P_{n} \equiv 0 \bmod 8$. Since $\lim _{3} k_{l}>\frac{1}{4} k^{*}, \lim _{3}{ }^{(0)} k_{1}>k^{*}$ and $\lim _{3}{ }^{(1)} k_{1}>k^{*}$ there are again only finitely many possibilities $(p, q)$.

(c) Consider $n \equiv l+1 \bmod 3$. Then $t$ must be at least 3 in case (i). Furthermore $\lim _{3} k_{t+1}=\frac{1}{9} k^{*}, \lim _{3}{ }^{(1)} k_{t+1}=k^{*}$, and $\lim _{3}{ }^{(i)} k_{t+1}$ and $\lim _{3}{ }^{(i)} k_{i+1}$ are greater than $k^{*}$ in all other cases. Thus we clearly have only finitely many possibilities for $(p, q)$ in this case when $k<k^{*}$. However if $k=k^{*}$ there are infinitely many possibilities for $(p, q)$ since $k_{3 m+1+4}<k^{*}$ whenever $k_{3 m+1+1}>k^{*}$.

To complete the proof of Theorem 3 it remains to prove

LemMA 15. Let $\alpha=\left[a_{0}, a_{1}, \cdots\right]>0$ be such that with $k=1.12$ equation (1) has only finitely many solutions $(p, q)$ such that neither $p$ nor $q$ is of the form $4^{a}(8 k+7)$. Then $\alpha$ satisfies the conditions of Lemma 14.

Proof. Before proceeding further it should be mentioned that no $\alpha$ satisfying the conditions of Lemma 15 actually exists, due to the fact that numbers congruent to 0 or $4 \bmod 8$ need not be of the form $4^{a}(8 k+7)$. It is precisely this fact that makes the reduction of $k=1.12$ to say $k=1$ extremely difficult in this approach to the problem. We first observe that if one of $p_{n}, q_{n}$ is of the form $4^{a}(8 k+7)$ then unless the other is of the form $2.4^{a}(8 k+7)$ the choice $(p, q)=\left(2 p_{n}, 2 q_{n}\right)$ has neither $p$ nor $q$ of the form $4^{a}(8 k+7)$. Secondly, for at least one of $t=1, t=2, t=3$ the choice $(p, q)=\left(t p_{n}, t q_{n}\right)$ has neither $p$ nor $q$ of the form $4^{a}(8 k+7)$. Consequently, if $\alpha$ is as in the statement of the lemma, then for all sufficiently large $n$ we must have

(i) At least one of $p_{n}, q_{n}$ is congruent to 0,4 or $7 \bmod 8$,

(ii) $\alpha_{n}+\alpha_{n}^{\prime} \leqq 9 / 1.12$, and hence $a_{n+1} \leqq 7$,

(iii) $\alpha_{n}+\alpha_{n}^{\prime} \leqq 4 / 1.12$, and hence $a_{n+1} \leqq 3$, unless one of $p_{n}, q_{n}$ is congruent to $7 \bmod 8$ and the other is congruent to 0 or $6 \bmod 8$. Considering $\left(P_{n}^{\prime}, Q_{n}^{\prime}\right)$ and $\left(P_{n}, Q_{n}\right)$ we also have for sufficiently large $n$ : 
(iv) At least one of $P_{n}, Q_{n}, P_{n-1}^{\prime}=p_{n+1}-p_{n}, Q_{n-1}^{\prime}=q_{n+1}-q_{n}$ must be congruent to 0,4 or $7 \bmod 8$ (since $\left.k_{n}^{\prime}+k_{n-1}^{\prime \prime}<2\right)$.

(v) If neither $p_{n+1}+p_{n}$ nor $q_{n+1}+q_{n}$ is congruent to 0,4 or $7 \bmod 8$ then $\left(p_{n+1}, q_{n+1}\right)$ must be one of $(0,7),(7,0),(6,7)$ or $(7,6) \bmod 8$. For otherwise we would have $a_{n+2} \leqq 3$, and $k_{n}^{\prime}<1.12$ unless $a_{n+2}=3, a_{n+1}=a_{n+3}=1$ and $a_{n} \geqq 5$. But then $4 k_{n+1}<1$ and $\left(2 p_{n+1}, 2 q_{n+1}\right)$ is valid.

(vi) Similarly if neither $p_{n+1}-p_{n}$ nor $q_{n+1}-q_{n}$ is congruent to 0,4 or 7 $\bmod 8$ then $\left(p_{n}, q_{n}\right)$ must be one of $(0,7),(7,0),(6,7)$ or $(7,6) \bmod 8$.

The remainder of the proof relies heavily on the above conditions. If $n$ is sufficiently large there are 236 possibilities for the 4-tuple $F_{n}=\left(p_{n}, q_{n}, p_{n+1}, q_{n+1}\right) \bmod 8$ satisfying (i) and the condition $p_{n} q_{n+1}-p_{n+1} q_{n} \equiv$ $\pm 1 \bmod 8$. Since the roles of $p$ and $q$ can be interchanged without affecting (i) to (vi) we really only have 118 possibilities to consider. These can be eliminated one by one till we are left only with the possibility that $\alpha$ satisfies the conditions of Lemma 14.

The elimination proceeds as follows.

(a) Ten possibilities for $F_{n}$ can be eliminated immediately because they do not transform by valid $a_{n+2}$ to valid $F_{n+1}$. These are $(0,1,1,0),(0,1,1,4)$, $(0,3,3,0),(0,5,3,0),(0,5,3,4),(1,4,4,7),(3,4,0,5),(4,5,5,0),(4,5,5,4)$ and $(4,7,7,2)$. We can now eliminate $(1,7,0,1),(1,7,4,3),(1,7,4,5),(2,7,1,4)$, $(3,7,0,3),(3,7,0,5)$ and $(4,7,7,4)$, followed by $(3,4,1,7),(4,5,7,3),(5,7,7,2)$ and $(6,7,7,4)$, since these transform by valid $a_{n+2}$ only into valid $F_{n+1}$ that have already been eliminated.

(b) Condition (iv) enables $(0,3,5,0),(0,5,5,0),(1,4,2,7),(1,4,4,1)$, $(2,7,7,4)$ and $(4,7,1,4)$ to be eliminated, and consequently $(0,7,1,4)$, $(1,7,2,7),(5,7,0,3),(5,7,4,7),(0,1,7,1),(0,7,7,5)$ and $(3,4,5,7)$ may be eliminated as in the second part of (a).

(c) Condition (v) enables $(0,3,3,7),(0,3,5,7),(0,5,5,4),(0,7,1,7)$, $(1,4,0,1),(1,7,5,4),(2,7,1,7),(2,7,3,7),(3,4,3,7),(3,4,7,1),(3,4,7,7)$, $(3,7,2,7),(3,7,3,4),(4,5,7,1),(4,5,7,5),(4,7,5,7),(5,7,5,4),(6,7,5,7)$, $(6,7,7,3),(6,7,7,7)$ and $(7,7,3,4)$ to be eliminated. In addition condition (vi) enables $(0,1,1,7),(0,3,3,4),(0,3,5,4),(0,5,3,7),(0,5,5,7),(1,4,7,5)$, $(1,7,3,4),(1,7,7,2),(2,7,7,1),(2,7,7,5),(3,4,4,5),(3,7,1,0),(3,7,5,4)$, $(4,5,1,7),(4,5,7,7),(4,7,1,0),(5,7,0,5),(5,7,3,4),(7,7,0,1)$ and $(7,7,4,5)$ to be eliminated. Consequently $(0,1,7,0),(0,1,7,2),(0,1,7,4),(0,7,7,2)$, $(0,7,7,3),(0,7,7,6),(3,4,0,3),(3,4,4,3),(4,5,3,0),(4,5,3,4)$ and $(5,7,4,1)$ may be eliminated.

(d) The following table lists in the first column as $H_{1}, \cdots, H_{32}$ the remaining thirty two possibilities for $F_{n}$. In the second column opposite $H_{i}$ the entry $H_{j}(k)$ indicates that if $F_{n}=H_{i}$ and $a_{n+2}=k$ then $F_{n+1}=H_{j}$. All such 
possibilities are listed. In the third column opposite $H_{i}$ the entry $H_{j}(k)$ indicates that if $F_{n-1}=H_{i}$ and $a_{n+1}=k$ then $F_{n}=H_{i}$.

Table 1

\begin{tabular}{|c|c|c|}
\hline$F_{n}$ & Transforms to $F_{n+1}\left(a_{n+2}\right)$ & Transforms from $F_{n-1}\left(a_{n+1}\right)$ \\
\hline$H_{1}=(0,7,1,0)$ & $H_{23}(3)$ & $H_{6}(4), H_{10}(7)$ \\
\hline$H_{2}=(0,7,7,0)$ & $H_{5}(1), H_{3}(4), H_{12}(7)$ & $H_{7}(4), H_{11}(7), H_{13}(2), H_{15}(6), H_{16}(1), H_{17}(3), H_{18}(5)$ \\
\hline$H_{3}=(0,7,7,4)$ & $H_{9}(1), H_{14}(2)$ & $H_{2}(4), H_{11}(3), H_{13}(6), H_{15}(2), H_{16}(5), H_{17}(7), H_{18}(1)$ \\
\hline$H_{4}=(0,1,7,7)$ & $H_{11}(1), H_{20}(2)$ & $H_{24}(2), H_{30}(1)$ \\
\hline$H_{s}=(0,7,7,7)$ & $H_{20}(1)$ & $H_{2}(1), H_{7}(5), H_{13}(3), H_{15}(7), H_{16}(2), H_{17}(4), H_{18}(6)$ \\
\hline$H_{6}=(1,4,0,7)$ & $H_{1}(4)$ & $H_{21}(1), H_{31}(2)$ \\
\hline$H_{7}=(4,7,7,0)$ & $H_{12}(3), H_{2}(4), H_{5}(5)$ & \\
\hline$H_{8}=(1,4,7,3)$ & $H_{17}(1)$ & $H_{31}(1)$ \\
\hline$H_{9}=(4,7,3,7)$ & $H_{19}(1)$ & $H_{3}(1)$ \\
\hline$H_{10}=(1,7,0,7)$ & $H_{1}(7)$ & $H_{12}(1)$ \\
\hline$H_{11}=(7,7,0,7)$ & $H_{3}(3), H_{12}(6), H_{2}(7)$ & $H_{\triangleleft}(1)$ \\
\hline$H_{12}=(0,7,7,1)$ & $H_{10}(1)$ & $H_{2}(7), H_{7}(3), H_{11}(6), H_{13}(1), H_{15}(5), H_{17}(2), H_{18}(4)$ \\
\hline$H_{13}=(2,7,7,0)$ & $H_{12}(1), H_{2}(2), H_{5}(3), H_{3}(6)$ & \\
\hline$H_{14}=(4,7,7,6)$ & $H_{15}(4)$ & $H_{3}(2), H_{22}(1)$ \\
\hline$H_{15}=(6,7,7,0)$ & $H_{3}(2), H_{12}(5), H_{2}(6), H_{5}(7)$ & $H_{14}(4), H_{32}(7)$ \\
\hline$H_{16}=(1,7,7,0)$ & $H_{2}(1), H_{s}(2), H_{3}(5)$ & \\
\hline$H_{17}=(3,7,7,0)$ & $H_{12}(2), H_{2}(3), H_{5}(4), H_{3}(7)$ & $H_{8}(1)$ \\
\hline$H_{18}=(5,7,7,0)$ & $H_{3}(1), H_{12}(4), H_{2}(5), H_{5}(6)$ & \\
\hline$H_{19}=(3,7,7,6)$ & $H_{30}(3), H_{31}(7)$ & $H_{4}(1), H_{23}(2)$ \\
\hline$H_{20}=(7,7,6,7)$ & $H_{31}(3), H_{30}(7)$ & $H_{4}(2), H_{5}(1)$ \\
\hline$H_{21}=(3,7,4,1)$ & $H_{6}(1)$ & \\
\hline$H_{22}=(3,7,4,7)$ & $H_{14}(1)$ & $H_{23}(1)$ \\
\hline$H_{23}=(0,1,7,3)$ & $H_{22}(1), H_{19}(2)$ & $H_{1}(3), H_{27}(2), H_{28}(1)$ \\
\hline$H_{24}=(0,1,7,5)$ & $H_{32}(1)$ & $H_{28}(1)$ \\
\hline$H_{25}=(0,1,7,6)$ & $H_{31}(4)$ & $H_{29}(1)$ \\
\hline$H_{26}=(1,4,6,7)$ & $H_{30}(4)$ & \\
\hline$H_{27}=(1,7,1,0)$ & $H_{23}(2)$ & \\
\hline$H_{28}=(2,7,1,0)$ & $H_{23}(1), H_{24}(3)$ & \\
\hline$H_{29}=(5,7,1,0)$ & $H_{25}(1), H_{4}(2)$ & \\
\hline$H_{30}=(6,7,1,0)$ & $H_{4}(1)$ & $H_{19}(3), H_{20}(7), H_{26}(4)$ \\
\hline$H_{31}=(6,7,1,4)$ & $H_{8}(1), H_{6}(2)$ & $H_{19}(7), H_{20}(3), H_{25}(4)$ \\
\hline$H_{32}=(5,7,6,7)$ & $H_{15}(7)$ & $H_{24}(1)$ \\
\hline
\end{tabular}

Clearly by assuming $n$ sufficiently large the transient states $H_{7}, H_{13}, H_{16}$, $H_{18}, H_{21}, H_{26}, H_{27}, H_{28}, H_{29}, H_{24}, H_{32}$ and $H_{25}$ may be eliminated.

(e) Consider the possibility $F_{n}=H_{10}$. Then $F_{n+1}=H_{1}$ and $F_{n+2}=H_{23}$. If $F_{n+3}=H_{22}$ then $k_{n+2}^{-1}>[3,2]+[0,8]$ contradicting (iii), so $F_{n+3}=H_{19}$. But now 


$$
k_{n+1}^{-1}>[0,1,2,1,1,8]+[7,3,2,7,1,1,2]>9 / 1.12
$$

contradicting (ii). Hence $H_{10}$, and consequently $H_{12}$, may be eliminated.

(f) Consider the possibility $F_{n}=H_{1}$. We must have

$$
k_{n+1}^{-1}>[3,2,3,2]+[0,4,2,8]>4 / 1.12
$$

contradicting (iii). Thus $H_{1}$ may be eliminated, followed by $H_{6}, H_{23}$ and $H_{22}$.

(g) Consider the possibility $F_{n}=H_{19}$. We cannot have $F_{n+1}=H_{31}$ for then

$$
k_{n+1}^{-1}=[7,1,1, \cdots]+[0,1,1, \cdots]>9 / 1.12
$$

contrary to (ii). Hence $F_{n+1}=H_{30}$ and $(p, q)=\left(P_{n}^{\prime}, Q_{n}^{\prime}\right)$ is valid. Since $k_{n}^{\prime \prime}$ is an increasing function of $\alpha_{n+1}$ and $\alpha_{n+1}^{\prime}$ we have

$$
k_{n}^{\prime \prime}<[1,1,2,3,2][2,1,1,2] /([3,1,2,3,2]+[0,1,1,2])<1.1 .
$$

Thus, for sufficiently large $n$ we may eliminate $H_{19}$ and consequently $H_{9}$.

(h) Consider the possibility $F_{n}=H_{14}$. Then $(p, q)=\left(P_{n}, Q_{n}\right)$ is valid and since $k_{n}^{\prime}$ is an increasing function of $\alpha_{n+1}$ and $\alpha_{n+1}^{\prime}$ we have

$$
k_{n}^{\prime}<[3,2,2,4][1,2,8] /([4,2,2,4]+[0,2,8])<1.1 \text {. }
$$

Hence for sufficiently large $n$ we may eliminate $H_{14}$ and consequently $H_{3}$ and the transient state $H_{15}$. But now $k_{n+1}^{-1}=[7,1, \cdots]+[0,1, \cdots]>9 / 1.12$ if $F_{n}=H_{11}$ so we may also eliminate $H_{11}$.

(j) Consider the possibility that $F_{n}=H_{20}$ and suppose that $F_{n+1}=H_{31}$. Then $(p, q)=\left(P_{n}^{\prime}, Q_{n}^{\prime}\right)$ is valid and

$$
k_{n}^{\prime \prime}<[1,1,1,3,2][2,1,5] /([3,1,1,3,2]+[0,1,5])<1.1 .
$$

This is impossible for sufficiently large $n$, so for large $n$ we have that if $F_{n}=H_{20}$ then $F_{n+1}=H_{30}$. But then we may eliminate $H_{31}, H_{8}, H_{17}, H_{2}$ and $H_{5}$ as being transient. We are now left only with the possibility that the conditions of Lemma 14 are satisfied, for $H_{4}, H_{20}$ and $H_{30}$ are the only remaining possibilities.

\section{References}

W. T. Scott (1940), 'Approximations to real irrationals by certain classes of rational fractions', Bull. Am. Math. Soc. 46 124-29.

C. A. Lekkerkerker (1969), Geometry of Numbers, Walters Nordhoff.

S. Lang (1966), Introduction to diophantine approximation, Addison Wesley.

Department of Mathematics,

Monash University,

Victoria 3168, Australia. 